\title{
An optimal endpoint trace embedding
}

\author{
Andrea Cianchi \\ Dipartimento di Matematica e Applicazioni per l'Architettura \\ Università di Firenze \\ Piazza Ghiberti 27, 50122 Firenze (Italy) \\ e-mail: cianchi@unifi.it \\ Luboš Pick \\ Department of Mathematical Analysis \\ Faculty of Mathematics and Physics, Charles University \\ Sokolovská 83, 18675 Praha 8 (Czech Republic) \\ e-mail: pick@karlin.mff.cuni.cz
}

\begin{abstract}
We find an optimal Sobolev-type space on $\mathbb{R}^{n}$ all of whose functions admit a trace on subspaces of $\mathbb{R}^{n}$ of given dimension. A corresponding trace embedding theorem with sharp range is established.
\end{abstract}

\section{Introduction and results}

One important property enjoyed by functions from the Sobolev space $W^{m, p}\left(\mathbb{R}^{n}\right), m \in \mathbb{N}$, $p \in[1, \infty]$, is that their restrictions, called traces, to lower dimensional spaces can be properly defined, provided that the dimension $d$ of the relevant subspaces is not too small, depending on $n, m$ and $p$. The trace of a function $u \in W^{m, p}\left(\mathbb{R}^{n}\right)$ turns out to be measurable with respect to the $d$-dimensional measure on the relevant subspaces, and also integrable to some power $q$, depending on $n, m, p$ and $d$. Loosely speaking, increasing the values of $m$ and $p$ causes $u$ to be more regular, and hence allows smaller values of $d$ and larger values of $q$.

To be more specific, let $n, d \in \mathbb{N}$, and let $n \geq 2$ and $1 \leq d<n$. Since $\mathbb{R}^{n}=\mathbb{R}^{d} \times \mathbb{R}^{n-d}$, any point $x \in \mathbb{R}^{n}$ can be represented as $x=(y, z)$, with $y \in \mathbb{R}^{d}$ and $z \in \mathbb{R}^{n-d}$. Moreover, $\mathbb{R}^{d}$ can be identified with the subspace of those points in $\mathbb{R}^{n}$ having the form $(y, 0)$ for some $y \in \mathbb{R}^{d}$. Given any $m \in \mathbb{N}$ and $p \in[1, \infty]$, the classical Sobolev space $W^{m, p}\left(\mathbb{R}^{n}\right)$ is defined as

$$
\begin{array}{r}
W^{m, p}\left(\mathbb{R}^{n}\right)=\left\{u: u \text { is an } m \text {-times weakly differentiable function on } \mathbb{R}^{n}\right. \\
\text { and } \left.\left|\nabla^{k} u\right| \in L^{p}\left(\mathbb{R}^{n}\right), 0 \leq k \leq m\right\} .
\end{array}
$$

Mathematics Subject Classification: 46E35, 46E30.

Keywords: Sobolev spaces, trace inequalities, Lorentz spaces, rearrangement invariant spaces.

This research was partially supported by the research project of MIUR "Partial differential equations and functional inequalities: quantitative aspects, geometric and qualitative properties, applications", by the Italian research project "Geometric properties of solutions to variational problems" of GNAMPA (INdAM) 2006, by the research project MSM 0021620839 of the Czech Ministry of Education, by grants 201/07/0388 and 201/08/0383 of the Grant Agency of the Czech Republic and by the Nečas Center for Mathematical Modeling project no. LC06052 financed by the Czech Ministry of Education. 
Here, $\nabla^{k} u$ stands for the vector of all partial derivatives of $u$ of order $k$, and $\left|\nabla^{k} u\right|$ denotes its length. The space $W_{\text {loc }}^{m, p}\left(\mathbb{R}^{n}\right)$ is defined with obvious modifications.

Various approaches to traces of functions are available in the literature. We shall adopt the following definition, which extends more customary notions of traces of functions in Sobolev spaces - see [Bu, Chapt. 5]. A function $u \in L_{\text {loc }}^{1}\left(\mathbb{R}^{n}\right)$ is said to have a trace $\operatorname{Tr} u \in L_{\text {loc }}^{1}\left(\mathbb{R}^{d}\right)$ on $\mathbb{R}^{d}$ if there exists a function $\bar{u}$, equivalent to $u$ on $\mathbb{R}^{n}$, such that

$$
\lim _{z \rightarrow 0} \bar{u}(\cdot, z)=\operatorname{Tr} u(\cdot) \quad \text { in } L_{\mathrm{loc}}^{1}\left(\mathbb{R}^{d}\right) .
$$

A standard trace embedding theorem (see e.g. [Ad, Theorem 5.4] or [Ma2, Corollary 1.4.1]), combined with [Bu, Cor. 1, Chapt. 5], tells us that if $1 \leq m<n$ and either

$$
d \geq n-m \text { and } p \geq 1,
$$

or

$$
d<n-m \text { and } p>\frac{n-d}{m},
$$

then every function $u \in W_{\text {loc }}^{m, p}\left(\mathbb{R}^{n}\right)$ has a trace on $\mathbb{R}^{d}$. Moreover, the operator $\operatorname{Tr}$, which associates $\operatorname{Tr} u$ with $u$, is linear, and, if $p<\frac{n}{m}$, then

$$
\operatorname{Tr}: W^{m, p}\left(\mathbb{R}^{n}\right) \rightarrow L^{\frac{d p}{n-m p}}\left(\mathbb{R}^{d}\right),
$$

where the arrow " $\rightarrow$ " stands for bounded operator. In particular,

$$
\|\operatorname{Tr} u\|_{L^{\frac{d p}{n-m p}\left(\mathbb{R}^{d}\right)}} \leq C\left\|\nabla^{m} u\right\|_{L^{p}\left(\mathbb{R}^{n}\right)}
$$

for every $u \in W^{m, p}\left(\mathbb{R}^{n}\right)$. Note that the case when $m \geq n$ is uninteresting, since any function $u \in W_{\mathrm{loc}}^{m, p}\left(\mathbb{R}^{n}\right), p \geq 1$, is continuous, and hence $\operatorname{Tr} u$ trivially exists on $\mathbb{R}^{d}$ for every $d \in$ $[1, n-1]$.

Unlike (1.1), in the limiting case when $p=\frac{n-d}{m}>1$, functions from the Sobolev space $W_{\text {loc }}^{m, \frac{n-d}{m}}\left(\mathbb{R}^{n}\right)$ need not admit a trace on $\mathbb{R}^{d}$.

The aim of this note is to fill in this gap and to exhibit an optimal (largest possible) Sobolev-type space when $d<n-m$ such that all of its functions admit a trace on $\mathbb{R}^{d}$. This is accomplished on calling into play the finer scale of Lorentz-Sobolev spaces. Indeed, we shall show that existence of traces can be restored when $p=\frac{n-d}{m}$, provided that $W_{\text {loc }}^{m, \frac{n-d}{m}}\left(\mathbb{R}^{n}\right)$ is replaced by the Sobolev type space $W_{\text {loc }}^{m} L^{\frac{n-d}{m}, 1}\left(\mathbb{R}^{n}\right)$ built upon the Lorentz space $L^{\frac{n-d}{m}}, 1\left(\mathbb{R}^{n}\right)$. Such a space is slightly smaller than $W_{\text {loc }}^{m, \frac{n-d}{m}}\left(\mathbb{R}^{n}\right)$ if $\frac{n-d}{m}>1$, but agrees with the standard space $W_{\text {loc }}^{m, 1}\left(\mathbb{R}^{n}\right)$ when $\frac{n-d}{m}=1$. Moreover, $L_{\text {loc }}^{\frac{n-d}{m}, 1}\left(\mathbb{R}^{n}\right)$ is optimal among all rearrangementinvariant spaces.

We recall that a rearrangement-invariant (r.i. for short) space $X\left(\mathbb{R}^{n}\right)$ is a Banach function space (in the sense of Luxemburg) of real-valued measurable functions in $\mathbb{R}^{n}$ endowed with a norm $\|\cdot\|_{X\left(\mathbb{R}^{n}\right)}$ satisfying

$$
\|u\|_{X\left(\mathbb{R}^{n}\right)}=\|v\|_{X\left(\mathbb{R}^{n}\right)} \quad \text { if } \quad u^{*}=v^{*} .
$$

Here, $u^{*}:(0, \infty) \rightarrow(0, \infty)$ denotes the decreasing rearrangement of $u$, namely

$$
u^{*}(s)=\sup \left\{t \geq 0: ? \mathcal{L}^{n}\left(\left\{x \in \mathbb{R}^{n}:|u(x)|>t\right\}\right)>s\right\} \quad \text { for } ? s \in(0, \infty),
$$

where $\mathcal{L}^{n}$ denotes the Lebesgue measure in $\mathbb{R}^{n}$. The representation space $\bar{X}(0, \infty)$ of an r.i. space $X\left(\mathbb{R}^{n}\right)$ is the r.i. space on $(0, \infty)$ equipped with the norm $\|\cdot\|_{\bar{X}(0, \infty)}$ fulfilling

$$
\|u\|_{X\left(\mathbb{R}^{n}\right)}=\left\|u^{*}\right\|_{\bar{X}(0, \infty)}
$$


for every $u \in X\left(\mathbb{R}^{n}\right)$.

The $m$-th order Sobolev space associated with $X\left(\mathbb{R}^{n}\right)$ is defined as

$$
\begin{array}{r}
W^{m} X\left(\mathbb{R}^{n}\right)=\left\{u: u \text { is an } m \text {-times weakly differentiable function on } \mathbb{R}^{n}\right. \\
\text { and } \left.\left|\nabla^{k} u\right| \in X\left(\mathbb{R}^{n}\right), 0 \leq k \leq m\right\},
\end{array}
$$

and is equipped with the norm $\|u\|_{W^{m} X\left(\mathbb{R}^{n}\right)}=\sum_{k=0}^{m}\left\|\nabla^{k} u\right\|_{X\left(\mathbb{R}^{n}\right)}$. The spaces $X_{\text {loc }}\left(\mathbb{R}^{n}\right)$ and $W_{\text {loc }}^{m} X\left(\mathbb{R}^{n}\right)$ are defined accordingly.

Given any $1<p<\infty$ and $1 \leq q \leq \infty$, the Lorentz space $L^{p, q}\left(\mathbb{R}^{n}\right)$ is the r.i. space of all measurable functions in $\mathbb{R}^{n}$ for which the quantity

$$
\|u\|_{L^{p, q}\left(\mathbb{R}^{n}\right)}=\left\|s^{\frac{1}{p}-\frac{1}{q}} u^{*}(s)\right\|_{L^{q}(0, \infty)}
$$

is finite. The functional $\|\cdot\|_{L^{p, q}\left(\mathbb{R}^{n}\right)}$ is always equivalent to an r.i. norm.

Observe that, since $L^{p, q}\left(\mathbb{R}^{n}\right) \subsetneq L^{p, r}\left(\mathbb{R}^{n}\right)$ if $1 \leq q<r \leq \infty$, and $L^{p, p}\left(\mathbb{R}^{n}\right)=L^{p}\left(\mathbb{R}^{n}\right)$, actually

$$
W^{m} L^{\frac{n-d}{m}, 1}\left(\mathbb{R}^{n}\right) \subsetneq W^{m, \frac{n-d}{m}}\left(\mathbb{R}^{n}\right) \quad \text { if } \frac{n-d}{m}>1,
$$

whereas

$$
W^{m} L^{1,1}\left(\mathbb{R}^{n}\right)=W^{m, 1}\left(\mathbb{R}^{n}\right) .
$$

Relations (1.8) and (1.9) continue to hold if all the Sobolev spaces are replaced by their local versions.

Theorem 1.1 Assume that $n \geq 2,1 \leq m<n$ and $1 \leq d \leq n-m$. Then any function from $W_{\mathrm{loc}}^{m} L^{\frac{n-d}{m}, 1}\left(\mathbb{R}^{n}\right)$ admits a trace on $\mathbb{R}^{d}$. Moreover, $L_{\mathrm{loc}}^{\frac{n-d}{m}, 1}\left(\mathbb{R}^{n}\right)$ is the optimal (largest) rearrangement-invariant space enjoying this property, in the sense that if $X\left(\mathbb{R}^{n}\right)$ is another r.i. space such that any function from $W_{\mathrm{loc}}^{m} X\left(\mathbb{R}^{n}\right)$ admits a trace on $\mathbb{R}^{d}$, then, necessarily, $X_{\mathrm{loc}}\left(\mathbb{R}^{n}\right) \subset L_{\mathrm{loc}}^{\frac{n-d}{m}}, 1\left(\mathbb{R}^{n}\right)$.

In an analogy with the classical situation described in (1.3)-(1.4), we establish a trace embedding for $W^{m} L^{\frac{n-d}{m}}, 1\left(\mathbb{R}^{n}\right)$. In fact, we find the optimal (smallest) range space in the class of Lorentz spaces for trace embeddings of $W^{m} L^{\frac{n-d}{m}, 1}\left(\mathbb{R}^{n}\right)$. Interestingly enough, the optimal range space in this endpoint trace embedding turns out not to be the genuine Lorentz space $L^{\frac{n-d}{m}, 1}\left(\mathbb{R}^{d}\right)$ as one would expect in the light of other known optimal Sobolev embeddings such as those treated in [On1, Pe, BW, Ha] (see [CP, EKP] for the optimality), and trace embeddings ([CKP]), but merely the (strictly larger) Lebesgue space $L^{\frac{n-d}{m}}\left(\mathbb{R}^{d}\right)$.

Theorem 1.2 Assume that $n \geq 2,1 \leq m<n$ and $1 \leq d \leq n-m$. Then

$$
\operatorname{Tr}: W^{m} L^{\frac{n-d}{m}, 1}\left(\mathbb{R}^{n}\right) \rightarrow L^{\frac{n-d}{m}}\left(\mathbb{R}^{d}\right) .
$$

In particular, a constant $C=C(n, m, d)$ exists such that

$$
\|\operatorname{Tr} u\|_{L^{\frac{n-d}{m}\left(\mathbb{R}^{d}\right)}} \leq C\left\|\nabla^{m} u\right\|_{L^{\frac{n-d}{m}, 1}\left(\mathbb{R}^{n}\right)}
$$

for every $u \in W^{m} L^{\frac{n-d}{m}, 1}\left(\mathbb{R}^{n}\right)$. Moreover, $L^{\frac{n-d}{m}}\left(\mathbb{R}^{d}\right)$ is optimal on the left-hand side of (1.11) among all Lorentz spaces.

Let us mention that a result on a related topic has recently been established in [CM], where a characterization of Sobolev inequalities involving general measures and Lorentz norms is given in terms of capacitary inequalities.

Remark 1.3 Embedding (1.10) continues to hold provided that the whole of $\mathbb{R}^{n}$ is replaced by any extension domain $\Omega$ (see e.g. [Ad, Bu, St1, Zi] for a definition). Of course, $\mathbb{R}^{d}$ has to be replaced by $\Omega \cap \mathbb{R}^{d}$ in this case.

Another generalization of embedding (1.10) concerns the case when traces on $d$-dimensional subspaces are replaced by (suitably defined - see e.g. [Bu]) traces on smooth $d$-dimensional Riemannian submanifolds of $\mathbb{R}^{n}$. 


\section{Proofs}

We begin with the proof of Theorem 1.2, to which the first part of the proof of Theorem 1.1 will be reduced. The proof of Theorem 1.2 involves two main ingredients: a sharp endpoint Sobolev inequality into the space of essentially bounded functions, and a boundedness result for integral product operators between Lorentz spaces.

The relevant Sobolev inequality is a refinement of a classical result and involves a LorentzSobolev space. It states that, if $1 \leq m<n$, then a constant $C=C(n, m)$ exists such that

$$
\|u\|_{L^{\infty}\left(\mathbb{R}^{n}\right)} \leq C\left\|\nabla^{m} u\right\|_{L^{\frac{n}{m}, 1}\left(\mathbb{R}^{n}\right)}
$$

for every $u \in W^{m} L^{\frac{n}{m}, 1}\left(\mathbb{R}^{n}\right)$. We are not able to trace back to the original proof of inequality (2.1), although it is certainly related to a result of [St2]. Anyway, inequality (2.1) with $m=1$ can be found in $[\mathrm{Ta}]$ and $[\mathrm{CP}]$. The case when $m>1$ can be derived from this one, via a (sub-limiting) Sobolev inequality in Lorentz spaces, which tells us that

$$
\|\nabla u\|_{L^{n, 1}\left(\mathbb{R}^{n}\right)} \leq C\left\|\nabla^{m} u\right\|_{L^{\frac{n}{m}, 1}\left(\mathbb{R}^{n}\right)}
$$

for every $u \in W^{m} L^{\frac{n}{m}}, 1\left(\mathbb{R}^{n}\right)([\mathrm{On} 1, \mathrm{Pe}])$.

The integral operators coming into play in our approach have the form

$$
h(y)=\int_{0}^{\infty} f(y, s) g(s) d s
$$

for measurable functions $f: \mathbb{R}^{d} \times(0, \infty) \rightarrow \mathbb{R}$ and $g:(0, \infty) \rightarrow \mathbb{R}$. A special case of [On2, Theorem C] ensures that if $p \in(1, \infty), f \in L^{p, 1}\left(\mathbb{R}^{d} \times(0, \infty)\right)$ and $g \in L^{p^{\prime}, \infty}(0, \infty)$, then $h \in L^{p}\left(\mathbb{R}^{d}\right)$, and a constant $C=C(p)$ exists such that

$$
\|h\|_{L^{p}\left(\mathbb{R}^{d}\right)} \leq C\|f\|_{L^{p, 1}\left(\mathbb{R}^{d} \times(0, \infty)\right)}\|g\|_{L^{p^{\prime}, \infty}(0, \infty)} .
$$

Proof of Theorem 1.2 We shall prove that a constant $C$ exists such that

$$
\|u(y, 0)\|_{L^{\frac{n-d}{m}}\left(\mathbb{R}^{d}\right)} \leq C\left\|\nabla^{m} u\right\|_{L^{\frac{n-d}{m}, 1}\left(\mathbb{R}^{n}\right)}
$$

for every $u \in W^{m} L^{\frac{n-d}{m}, 1}\left(\mathbb{R}^{n}\right) \cap C_{0}^{\infty}\left(\mathbb{R}^{n}\right)$. Since this space is dense in $W^{m} L^{\frac{n-d}{m}, 1}\left(\mathbb{R}^{n}\right)$, as shown by a standard convolution argument, it will follow via [Bu, Cor. 1, Chapt. 5] that the operator $\operatorname{Tr}$ is well defined in $W^{m} L^{\frac{n-d}{m}, 1}\left(\mathbb{R}^{n}\right)$ and that (1.10)-(1.11) hold.

Fix any $u$ as above. Then,

$$
\left|\nabla^{m} u\right| \in L^{\frac{n-d}{m}, 1}\left(\mathbb{R}^{n}\right)
$$

Consider the function $U: \mathbb{R}^{d} \times(0, \infty) \rightarrow[0, \infty)$ given by

$$
U(y, s)=\left|\nabla^{m} u\right|(y, \cdot)^{*}(s) \quad \text { for }(y, s) \in \mathbb{R}^{d} \times(0, \infty) .
$$

It is easily seen, as a consequence of Fubini's theorem, that

$$
\mathcal{L}^{d+1}\left(\left\{(y, s) \in \mathbb{R}^{d} \times(0, \infty): U(y, s)>t\right\}\right)=\mathcal{L}^{n}\left(\left\{x \in \mathbb{R}^{n}:\left|\nabla^{m} u(x)\right|>t\right\}\right) \quad \text { for } t>0 .
$$

Hence,

$$
U^{*}=\left|\nabla^{m} u\right|^{*}
$$

By (2.4) and (2.5),

$$
U \in L^{\frac{n-d}{m}, 1}\left(\mathbb{R}^{d} \times(0, \infty)\right)
$$


Inequality (2.1), with $n-d$ in place of $n$, entails that

$$
\|w\|_{L^{\infty}\left(\mathbb{R}^{n-d}\right)} \leq C\left\|\nabla^{m} w\right\|_{L^{\frac{n-d}{m}, 1}\left(\mathbb{R}^{n-d}\right)}
$$

for some constant $C=C(n, m, d)$ and for every $w \in W^{m} L^{\frac{n-d}{m}, 1}\left(\mathbb{R}^{n-d}\right)$. From (2.7), applied to $w(\cdot)=u(y, \cdot)$ for each $y \in \mathbb{R}^{d}$, we deduce that,

$$
\begin{aligned}
|u(y, 0)| & \leq\|u(y, \cdot)\|_{L^{\infty}\left(\mathbb{R}^{n-d}\right)} \leq C\left\|\nabla_{z}^{m} u(y, \cdot)\right\|_{L^{\frac{n-d}{m}, 1}\left(\mathbb{R}^{n-d}\right)} \\
& \leq C\left\|\nabla^{m} u(y, \cdot)\right\|_{L^{\frac{n-d}{m}, 1}\left(\mathbb{R}^{n-d}\right)}=C \int_{0}^{\infty} U(y, s) s^{\frac{m}{n-d}-1} d s .
\end{aligned}
$$

Here, $\nabla_{z}^{m} u$ denotes the vector of all the derivatives of $u$ of order $m$ with respect to the $z$ variables. The function $s^{\frac{m}{n-d}-1}$ belongs to $L^{\frac{n-d}{n-d-m}, \infty}(0, \infty)$, and $\left\|s^{\frac{m}{n-d}-1}\right\|_{L^{\frac{n-d}{n-d-m}, \infty}(0, \infty)}=$ 1. Thus, owing to $(2.2)$ and (2.5), there exists a constant $C=C(n, m, d)$ such that

$$
\begin{aligned}
\left\|\int_{0}^{\infty} U(\cdot, s) s^{\frac{m}{n-d}-1} d s\right\|_{L^{\frac{n-d}{m}\left(\mathbb{R}^{d}\right)}} & \leq C\|U\|_{L^{\frac{n-d}{m}, 1}\left(\mathbb{R}^{d} \times(0, \infty)\right)}\left\|s^{\frac{m}{n-d}-1}\right\|_{L^{\frac{n-d}{n-d-m}, \infty}(0, \infty)} \\
& =C\left\|\nabla^{m} u\right\|_{L^{\frac{n-d}{m}, 1}\left(\mathbb{R}^{n}\right)} .
\end{aligned}
$$

Coupling (2.8) and (2.9) yields (2.3).

In order to demonstrate the sharpness of $L^{\frac{n-d}{m}}\left(\mathbb{R}^{d}\right)$ as a range space, we begin by observing that an inequality of the form

$$
\|\operatorname{Tr} u\|_{L^{p, q}\left(\mathbb{R}^{d}\right)} \leq C\left\|\nabla^{m} u\right\|_{L^{\frac{n-d}{m}, 1}\left(\mathbb{R}^{n}\right)}
$$

can hold for some $p$ and $q$ only if $p=\frac{n-d}{m}$. This follows by a scaling argument, on replacing $u(x)$ by $u(\lambda x)$ for $\lambda>0$.

Thus, it suffices to exhibit a function $u \in W^{m} L^{\frac{n-d}{m}, 1}\left(\mathbb{R}^{n}\right)$ such that, whenever $q \in\left[1, \frac{n-d}{m}\right)$, we have that $\operatorname{Tr} u \notin L^{\frac{n-d}{m}, q}\left(\mathbb{R}^{d}\right)$. We shall produce, in fact, a compactly supported function $u \in W^{m} L^{\frac{n-d}{m}, 1}\left(\mathbb{R}^{n}\right)$ such that $\operatorname{Tr} u \notin L_{\mathrm{loc}}^{\frac{n-d}{m}}, q\left(\mathbb{R}^{d}\right)$ for $q \in\left[1, \frac{n-d}{m}\right)$. Pick any number $\alpha$ such that

$$
1+\frac{m}{n-d}<\alpha \leq 1+\frac{1}{q}
$$

Let $\varphi:(0,1) \times(0,1) \rightarrow[0, \infty)$ be the function given by

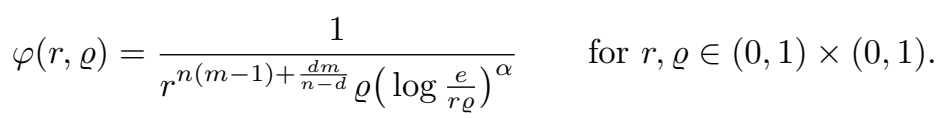

Define $u: \mathbb{R}^{n} \rightarrow[0, \infty)$ as

$$
u(y, z)=\int_{|z|^{n-d}}^{|y|^{n}} \varphi(|y|, \varrho)\left(|y|^{n}-\varrho\right)^{m-1} d t \quad \text { if }|z|^{n-d} \leq|y|^{n}<1,
$$

and $u(y, z)=0$ otherwise. We begin by showing that, given any $q \in\left[1, \frac{n-d}{m}\right)$,

$$
\operatorname{Tr} u \notin L_{\mathrm{loc}}^{\frac{n-d}{m}, q}\left(\mathbb{R}^{d}\right) .
$$

One has that

$$
\begin{aligned}
\operatorname{Tr} u(y) & =u(y, 0)=\int_{0}^{|y|^{n}} \varphi(|y|, \varrho)\left(|y|^{n}-\varrho\right)^{m-1} d \varrho \\
& \geq \frac{1}{2^{m-1}} \int_{0}^{\frac{|y|^{n}}{2}} \varphi(|y|, \varrho)|y|^{n(m-1)} d \varrho=C|y|^{-\frac{d m}{n-d}}\left(\log \frac{e}{|y|^{1+n}}\right)^{1-\alpha}
\end{aligned}
$$


for some constant $C$, if $|y| \leq 1$, and $\operatorname{Tr} u(y)=0$ otherwise. Thus, there exists a constant $C$ such that

$$
(\operatorname{Tr} u)^{*}(s) \geq C s^{-\frac{m}{n-d}}\left(\log \frac{e}{s}\right)^{1-\alpha} \text { for small } s,
$$

and (2.12) follows by the second inequality in (2.10).

We conclude by proving that $u \in W^{m} L^{\frac{n-d}{m}, 1}\left(\mathbb{R}^{n}\right)$. It is easily verified that $u$ is $m$-times weakly differentiable. Thus, since $u$ is compactly supported, thanks to a general Poincarétype inequality (see e.g. [CP, Lemma 4.2]), it suffices to show that

$$
\left|\nabla^{m} u\right| \in L^{\frac{n-d}{m}, 1}\left(\mathbb{R}^{n}\right) .
$$

An induction argument on the order of differentiation yields the following estimate for the norm of the vector $\nabla_{y}^{m} u$ of all partial derivatives of $u$, of order $m$, with respect to the $y$-variables only:

$$
\left|\nabla_{y}^{m} u\right| \leq C|y|^{-\frac{n m}{n-d}}\left(\log \frac{C}{|y|}\right)^{1-\alpha}
$$

for some constant $C$, if $|z|^{n-d} \leq|y|^{n}<1$, whereas $\nabla_{y}^{m} u$ vanishes otherwise. Thus, on defining $\zeta:(0,1] \rightarrow[0, \infty)$ as

$$
\zeta(s)=C s^{-\frac{m}{n-d}}\left(\log \frac{C}{s}\right)^{1-\alpha} \quad \text { for } s \in(0,1]
$$

with a suitable choice of $C$, one has that $\zeta$ is decreasing in $(0,1]$, and, for every $t>0$,

$$
\begin{aligned}
\mathcal{L}^{n}\left(\left\{(y, z) \in \mathbb{R}^{n}:\left|\nabla_{y}^{m} u\right|>t\right\}\right) & \leq \mathcal{L}^{n}\left(\left\{(y, z) \in \mathbb{R}^{n}:|z|^{n-d} \leq|y|^{n}<1, \zeta\left(|y|^{n}\right)>t\right\}\right) \\
& \leq \int_{\left\{|y| \leq\left(\zeta^{-1}(t)\right)^{1 / n}\right\}}\left(\int_{\left\{|z| \leq|y|^{n /(n-d)}\right\}} d z\right) d y \\
& =C^{\prime} \int_{\left\{|y| \leq\left(\zeta^{-1}(t)\right)^{1 / n}\right\}}|y|^{n} d y=C^{\prime \prime}\left(\zeta^{-1}(t)\right)^{\frac{d}{n}+1}
\end{aligned}
$$

for some constants $C^{\prime}$ and $C^{\prime \prime}$. Hence, there exists a constant $C$ such that

$$
\left|\nabla_{y}^{m} u\right|^{*}(s) \leq C s^{-\frac{n m}{(n+d)(n-d)}}\left(\log \frac{C}{s}\right)^{1-\alpha} \text { for small } s .
$$

Next, an induction argument again shows that the norm of the vector $\nabla_{y, z}^{m} u$ of all $m$-th order partial derivatives of $u$ involving also differentiation along the $z$ variables admits the bound:

$$
\left|\nabla_{y, z}^{m} u\right| \leq C|y|^{-\frac{d m}{n-d}}|z|^{-m}\left(\log \frac{C}{|y||z|}\right)^{-\alpha},
$$

for some constant $C$, if $|z|^{n-d} \leq|y|^{n}<1$, and vanishes otherwise. Hence, if we define $\sigma:(0,1] \rightarrow[0, \infty)$ as

$$
\sigma(s)=C s^{-\frac{m}{n-d}}\left(\log \frac{C}{s}\right)^{-\alpha} \quad \text { for } s \in(0,1],
$$


then, for a suitable choice of $C$, the function $\sigma$ is decreasing in $(0,1]$, and, for every $t>0$,

$$
\begin{aligned}
\mathcal{L}^{n}\left(\left\{(y, z) \in \mathbb{R}^{n}:\left|\nabla_{y, z}^{m} u\right|>t\right\}\right) \leq & \mathcal{L}^{n}\left(\left\{(y, z) \in \mathbb{R}^{n}:|z|^{n-d} \leq|y|^{n}<1, \sigma\left(|y|^{d}|z|^{n-d}\right)>t\right\}\right) \\
\leq & \mathcal{L}^{n}\left(\left\{(y, z) \in \mathbb{R}^{n}:|y| \leq 1,|z| \leq 1,|z|<\left(\frac{\sigma^{-1}(t)}{|y|^{d}}\right)^{1 /(n-d)}\right\}\right) \\
= & \int_{\left\{\left(\sigma^{-1}(t)\right)^{1 / d} \leq|y| \leq 1\right\}}\left(\int_{\left\{|z| \leq\left(\frac{\sigma^{-1}(t)}{\left.|y|\right|^{d}}\right)^{1 /(n-d)}\right\}} d z\right) d y \\
& +\int_{\left\{|y|<\left(\sigma^{-1}(t)\right)^{1 / d}\right\}}\left(\int_{\{|z| \leq 1\}} d z\right) d y \\
= & C^{\prime}\left(\int_{\left\{\left(\sigma^{-1}(t)\right)^{1 / d} \leq|y| \leq 1\right\}} \frac{\sigma^{-1}(t)}{|y|^{d}} d y+\int_{\left\{|y|<\left(\sigma^{-1}(t)\right)^{1 / d}\right\}} d y\right) \\
\leq & C^{\prime \prime}\left(\sigma^{-1}(t) \log \left(\frac{1}{\sigma^{-1}(t)}\right)+\sigma^{-1}(t)\right),
\end{aligned}
$$

for some constants $C^{\prime}$ and $C^{\prime \prime}$. Hence, there exists a constant $C$ such that

$$
\left|\nabla_{y, z}^{m} u\right|^{*}(s) \leq C s^{-\frac{m}{n-d}}\left(\log \frac{e}{s}\right)^{\frac{m}{n-d}-\alpha} \text { for small } s .
$$

From (2.16) and (2.18) one deduces that

$$
\left|\nabla^{m} u\right|^{*}(s) \leq C s^{-\frac{m}{n-d}}\left(\log \frac{e}{s}\right)^{\frac{m}{n-d}-\alpha} \text { for small } s,
$$

whence (2.14) follows.

We conclude with the proof of Theorem 1.1.

Proof of Theorem 1.1 Let $u \in W_{\mathrm{loc}}^{m} L^{\frac{n-d}{m}, 1}\left(\mathbb{R}^{n}\right)$. Then, given any ball $B$ centered on $\mathbb{R}^{d}$, we have that $u \in W^{m} L^{\frac{n-d}{m}, 1}(B)$. An extension theorem for Sobolev spaces built upon arbitrary r.i. spaces (see $[\mathrm{CR}]$ ) ensures that $u$ admits an extension $\widetilde{u}$ on $\mathbb{R}^{n}$ such that $\widetilde{u} \in W^{m} L^{\frac{n-d}{m}, 1}\left(\mathbb{R}^{n}\right)$. By Theorem 1.2, $\widetilde{u}$ admits a trace on $\mathbb{R}^{d}$, and hence, owing to the arbitrariness of $B, u$ admits a trace on $\mathbb{R}^{d}$ as well.

As far as the optimality of the space $W_{\text {loc }}^{m} L^{\frac{n-d}{m}, 1}\left(\mathbb{R}^{n}\right)$ is concerned, assume that $X\left(\mathbb{R}^{n}\right)$ is an r.i. space such that every function from $W_{\mathrm{loc}}^{m} X\left(\mathbb{R}^{n}\right)$ has a trace on $\mathbb{R}^{d}$. We have to show that

$$
X_{\mathrm{loc}}\left(\mathbb{R}^{n}\right) \subset L_{\mathrm{loc}}^{\frac{n-d}{m}, 1}\left(\mathbb{R}^{n}\right) .
$$

If $\frac{n-d}{m}=1$, then $L_{\text {loc }}^{\frac{n-d}{m}}, 1\left(\mathbb{R}^{n}\right)=L_{\text {loc }}^{1}\left(\mathbb{R}^{n}\right)$, and hence (2.19) holds as a consequence of a baisc property of r.i. spaces.

Assume now that $\frac{n-d}{m}>1$. We shall show that, if

$$
X_{\mathrm{loc}}\left(\mathbb{R}^{n}\right) \backslash L_{\mathrm{loc}}^{\frac{n-d}{m}}, 1\left(\mathbb{R}^{n}\right) \neq \emptyset,
$$

then there exists a function

$$
u \in W_{\mathrm{loc}}^{m} X\left(\mathbb{R}^{n}\right)
$$

such that, for every $v \in L_{\mathrm{loc}}^{1}\left(\mathbb{R}^{d}\right)$,

$$
\lim _{z \rightarrow 0}\|u(\cdot, z)-v(\cdot)\|_{L^{1}\left(B^{d}\right)}=\infty .
$$


Here, $B^{d}$ denotes the unit ball in $\mathbb{R}^{d}$ centered at 0 . By (2.20), there exists a decreasing function $f:(0, \infty) \rightarrow[0, \infty)$ such that

$$
f \chi_{\left(0, \omega_{n-d}\right)} \in \bar{X}(0, \infty)
$$

but

$$
f \chi_{\left(0, \omega_{n-d}\right)} \notin L^{\frac{n-d}{m}, 1}(0, \infty) .
$$

Here, $\omega_{n-d}$ denotes the measure of $B^{n-d}$. Define $w: B^{n-d} \rightarrow[0, \infty)$ as

$$
w(z)=\int_{\omega_{n-d}|z|^{n-d}}^{\omega_{n-d}} f(s) s^{-m\left(1-\frac{1}{n-d}\right)}\left(s-\omega_{n-d}|z|^{n-d}\right)^{m-1} d s,
$$

and $u: \mathbb{R}^{n} \rightarrow[0, \infty)$ as

$$
u(y, z)=\xi(y) \eta(z) w(z) \quad \text { for }(y, z) \in \mathbb{R}^{n},
$$

where $\xi \in C_{0}^{\infty}\left(\mathbb{R}^{d}\right), 0 \leq \xi \leq 1, \xi(y)=1$ for $y \in B^{d}$, and $\eta \in C_{0}^{\infty}\left(\mathbb{R}^{n-d}\right), 0 \leq \eta \leq 1, \eta(z)=1$ for $z \in B^{n-d}$. By (2.24),

$$
\lim _{z \rightarrow 0} w(z)=\infty
$$

and hence

$$
\lim _{z \rightarrow 0} u(y, z)=\infty
$$

uniformly as $y \in B^{d}$. Thus, (2.22) follows.

We next prove (2.21). One can easily verify that

$$
\left|\nabla^{m} u(y, z)\right| \leq \phi(y) \psi(z)\left|\nabla_{z}^{m} w(z)\right| \quad \text { for }(y, z) \in \mathbb{R}^{n},
$$

for some nonnegative functions $\phi \in C_{0}^{\infty}\left(\mathbb{R}^{d}\right)$ and $\psi \in C_{0}^{\infty}\left(\mathbb{R}^{n-d}\right)$. Define $g:(0, \infty) \rightarrow[0, \infty)$ as

$$
g(s)=\sum_{i=1}^{m-1} s^{i-\frac{m}{n-d}} \int_{s}^{\infty} \chi_{\left(0, \omega_{n-d}\right)}(r) f(r) r^{-i+\frac{m}{n-d}-1} d r \quad \text { for } s>0,
$$

and $h:(0, \infty) \rightarrow[0, \infty)$ as

$$
h=f+g .
$$

An induction argument on the order of differentiation shows that there exists a constant $C$ such that

$$
\left|\nabla_{z}^{m} w(z)\right| \leq C h\left(\omega_{n-d}|z|^{n-d}\right) \quad \text { if } z \in B^{n-d},
$$

and $\nabla_{z}^{m} w(z)=0$ otherwise. Thus, there exist constants $C$ and $C^{\prime}$ such that

$$
\begin{aligned}
\mathcal{L}^{n}\left(\left\{x \in \mathbb{R}^{n}:\left|\nabla^{m} u\right|>t\right\}\right) & \leq \mathcal{L}^{n}\left(\left\{(y, z) \in \mathbb{R}^{n}: \phi(y) \psi(z) h\left(\omega_{n-d}|z|\right)>t\right\}\right) \\
& \leq \mathcal{L}^{n}\left(\left\{(y, z) \in \operatorname{supp} \phi \times \operatorname{supp} \psi: C h\left(\omega_{n-d}|z|\right)>t\right\}\right) \\
& \leq C^{\prime} \mathcal{L}^{n-d}\left(\left\{z \in \mathbb{R}^{n-d}: C h\left(\omega_{n-d}|z|\right)>t\right\}\right)
\end{aligned}
$$

for $t>0$. Hence, a constant $C$ exists such that

$$
\begin{aligned}
\left|\nabla^{m} u\right|^{*}(s) & \leq C\left(h \chi_{\left(0, \omega_{n-d}\right)}\right)^{*}(s / C) \\
& \leq C\left(\left(f \chi_{\left(0, \omega_{n-d}\right)}\right)^{*}(s /(2 C))+\left(g \chi_{\left(0, \omega_{n-d}\right)}\right)^{*}(s /(2 C))\right) \quad \text { for } s>0 .
\end{aligned}
$$

Note that in the last inequality we have made use of the fact that, by a property of the operation of decreasing rearrangement, $(f+g)^{*}(s) \leq f^{*}(s / 2)+g^{*}(s / 2)$ for $s>0$ (see [BS, 
Chapter 2, Proposition 1.7]). By (2.29) and by the boundedness of the dilation operator in any rearrangement invariant space (see [BS, Chapter 3, Proposition 5.11]),

$$
\left\|\nabla^{m} u\right\|_{X\left(\mathbb{R}^{n}\right)}=\left\|\left|\nabla^{m} u\right|^{*}\right\|_{\bar{X}(0, \infty)} \leq C\left(\left\|f \chi_{\left(0, \omega_{n-d}\right)}\right\|_{\bar{X}(0, \infty)}+\left\|g \chi_{\left(0, \omega_{n-d}\right)}\right\|_{\bar{X}(0, \infty)}\right)
$$

for some constant $C$. On the other hand, it is easy to see that the Hardy-type operators appearing on the right-hand side of $(2.26)$ are bounded on $L^{1}(0, \infty)$ and on $L^{\infty}(0, \infty)$, and hence, by an interpolation theorem of Calderón ([BS, Chapter 3, Theorem 2.12]), they are also bounded on any rearrangement invariant space on $(0, \infty)$. Hence, $\|g\|_{\bar{X}(0, \infty)} \leq$ $C\left\|f \chi_{\left(0, \omega_{n-d}\right)}\right\|_{\bar{X}(0, \infty)}<\infty$ for some constant $C$, and (2.21) follows from (2.30) and (2.23).

\section{References}

[AH] D.R.Adams \& L.I.Hedberg, "Function spaces and potential theory", Springer, Berlin, 1996.

[Ad] R.A.Adams, "Sobolev spaces", Academic Press, Orlando, 1975. Hardy-Sobolev inequality and its applications, $W^{1, p}$ and its applications to Shrödinger operators, Lorentz spaces,

[BS] C.Bennett \& R.Sharpley, "Interpolation of operators", Academic Press, Boston, 1988. 5 (1930), 40-46. 38

[BW] H.Brézis \& S.Wainger, A note on limiting cases of Sobolev embeddings and convolution inequalities, Comm. Part. Diff. Eq. 5 (1980), 773-789.

[Bu] V.Burenkov, "Sobolev spaces on domains", Teubner, Stuttgart, 1998.

[CP] A.Cianchi \& L.Pick, Sobolev embeddings into BMO, VMO and $L^{\infty}$, Arkiv Mat. 36 (1998), 317-340.

[CKP] A.Cianchi, R.A.Kerman \& L.Pick, Boundary trace inequalities and rearrangements, J. Anal. Math. 105 (2008), 241-265.

[CR] A.Cianchi \& M.Randolfi, The sharp modulus of continuity of Orlicz-Sobolev functions, preprint.

[CM] S.Costea \& V.G.Maz'ya, Conductor inequalities and criteria for Sobolev-Lorentz twoweight inequalities, preprint.

[EKP] D.E.Edmunds, R.A.Kerman \& L.Pick, Optimal Sobolev imbeddings involving rearrangement invariant quasi-norms, J. Funct. Anal. 170 (2000), 307-355.

[Ha] K.Hansson, Imbedding theorems of Sobolev type in potential theory, Math. Scand. 45 (1979), 77-102.

[Ma2] V.G.Maz'ya, “Sobolev spaces”, Springer-Verlag, Berlin, 1985.

[On1] R.O'Neil, Convolution operators in L $(p, q)$ spaces, Duke Math. J. 30 (1963), 129-142.

[On2] R. O'Neil, Integral transforms and tensor products on Orlicz spaces and $L(p, q)$ spaces, J. Anal. Math. 21 (1968), 1-276.

[Pe] J.Peetre, Espaces d' interpolation et théorème de Soboleff, Ann. Inst. Fourier 16 (1966), 279-317.

[St1] E.M.Stein, "Singular integrals and differentiablity properties of functions", Princeton University Press, Princeton, 1970.

[St2] E.M.Stein, The differentiability of functions in $\mathbb{R}^{n}$, Ann. Math. 113 (1981), 383-385.

[Ta] G.Talenti, Inequalities in rearrangement invariant function spaces, in Nonlinear analysis, function spaces and applications, Vol. 5, M.Krbec, A.Kufner, B.Opic and J.Rákosnik Eds., Prometheus Publishing House, Prague, 1994.

[Zi] W.P.Ziemer, "Weakly differentiable functions", Spriger-Verlag, New York, 1989. 